\title{
Erratum to: Overexpression of Human E46K Mutant $\alpha$-Synuclein Impairs Macroautophagy via Inactivation of JNK1-Bcl-2 Pathway
}

\author{
Jia-Qing Yan • Yu-He Yuan • Yan-Na Gao • \\ Ju-Yang Huang • Kai-Li Ma • Yan Gao • \\ Wan-Qing Zhang $\cdot$ Xiao-Feng Guo $•$ Nai-Hong Chen
}

Published online: 1 June 2014

(C) Springer Science+Business Media New York 2014

\section{Erratum to: Mol Neurobiol}

DOI 10.1007/s12035-014-8738-1

The original version of the article unfortunately contains mistake on Fig. 2a as the upper image should be from the GFP group cells. This correction does not change the conclusion of the article. The authors are hereby correcting this figure as shown.

The online version of the original article can be found at http://dx.doi.org/ 10.1007/s12035-014-8738-1.

J.-Q. Yan • Y.-H. Yuan • Y.-N. Gao • J.-Y. Huang • K.-L. Ma •

Y. Gao $\cdot$ W.-Q. Zhang $\cdot$ N.-H. Chen $(\square)$

State Key Laboratory of Bioactive Substances and Functions of

Natural Medicines, Department of Pharmacology, Institute of

Materia Medica, Chinese Academy of Medical Sciences and Peking

Union Medical College, I Xiannongtan Street, Xicheng District,

100050 Beijing, People's Republic of China

e-mail: chennh@imm.ac.cn

Y.-N. Gao $\cdot$ W.-Q. Zhang

Tianjin University of Traditional Chinese Medicine, Tianjin,

People's Republic of China

Y.-N. Gao

Beijing Key Laboratory of New Drug Mechanisms and

Pharmacological Evaluation Study, Beijing,

People's Republic of China

X.-F. Guo

Shanxi University of Traditional Chinese Medicine, Taiyuan,

People's Republic of China 
a
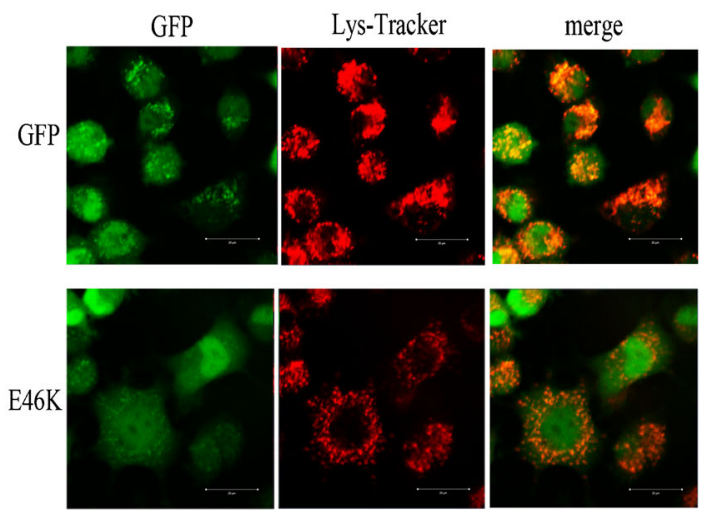

d
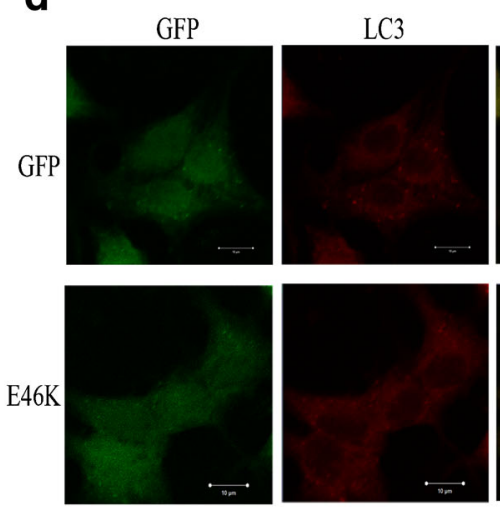

\section{$\mathbf{f}$}
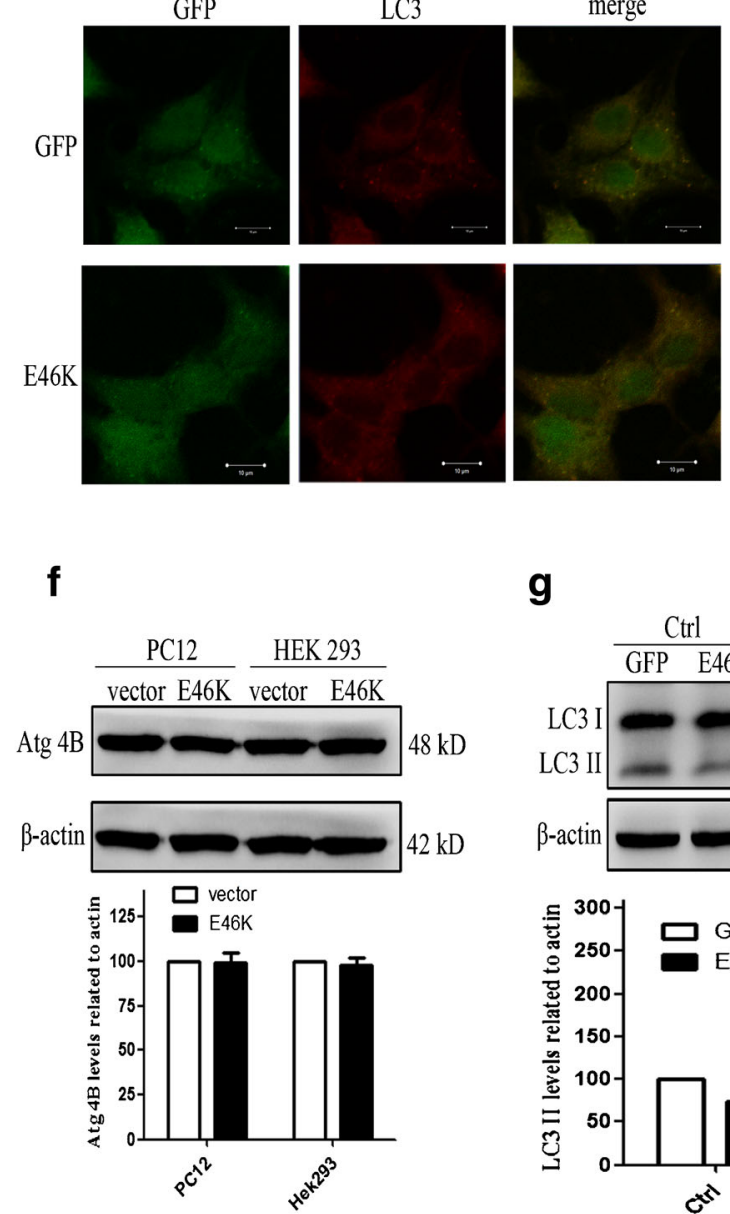

b
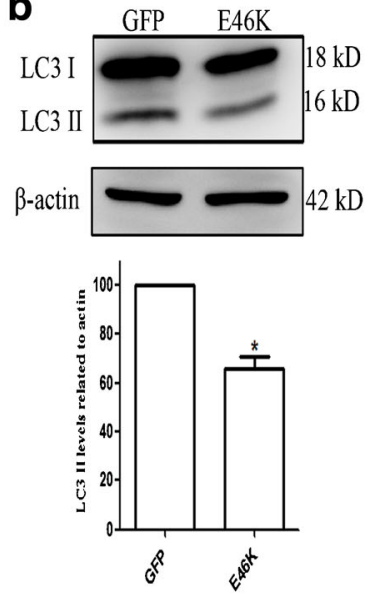

e

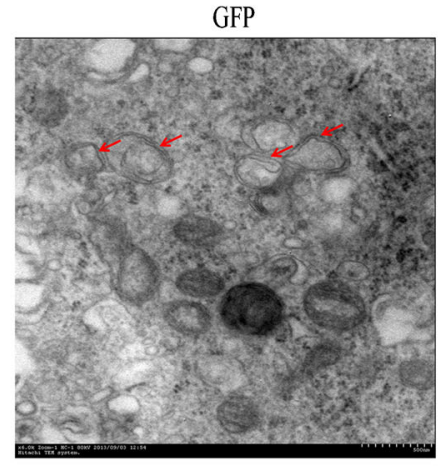

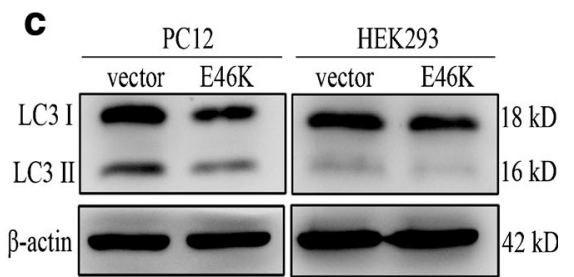
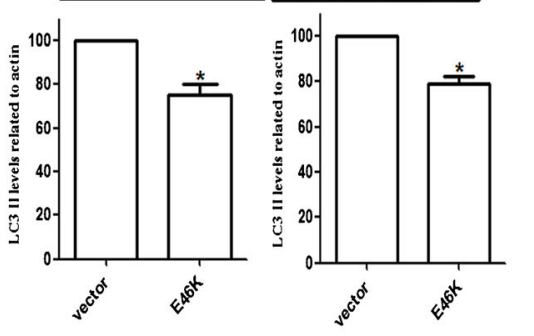

E46K

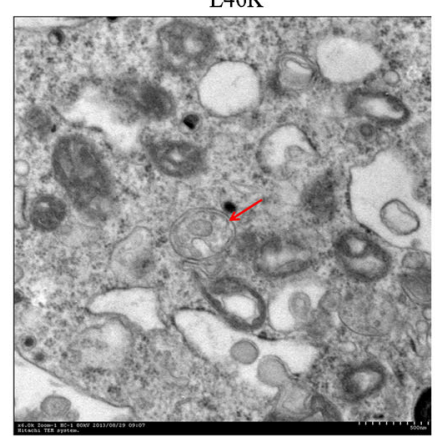

g
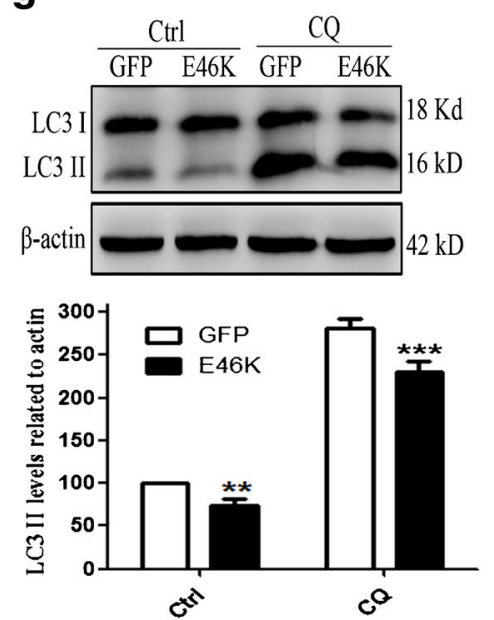

h

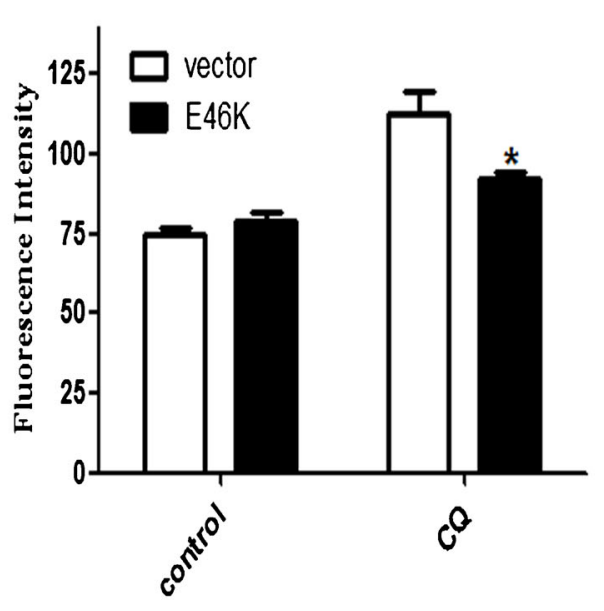

Fig. 2 Overexpression of E46K mutant $\alpha$-synuclein inhibits autophagy. induction. (a) PC12 cells stably overexpressing E46K mutant $\alpha$ synuclein GFP were labeled with $50 \mathrm{nM}$ of LysoTracker for $2 \mathrm{~h}$ and then visualized using a confocal microscopy. Scale bar $10 \mu \mathrm{m}$. PC12 cells stable overexpression of E46K mutant $\alpha$-synuclein GFP (b) or PC12 and HEK293 cells transfected with E46K mutant $\alpha$-synuclein (c) for $48 \mathrm{~h}$ were harvested, and protein extracts were immunoblotted for LC3. (d) Immunostaining of endogenous LC3 positive puncta in PC12 cells stably transfected with E46K mutant $\alpha$-synuclein GFP. Scale bar $10 \mu \mathrm{m}$. (e) Analysis of autophagosome (indicated with a red arrow) numbers in PC12 cells stably overexpressing E46K mutant $\alpha$-synuclein GFP by
EM. Scale bar $500 \mathrm{~nm}$. (f) PC12 cells stably and HEK293 cells transiently transfected with E46K mutant $\alpha$-synuclein were lysted and immunoblotted with an anti-Atg 4B antibody. (g) PC12 cells stably overexpressing E46K mutant $\alpha$-synuclein GFP were treated with and without $100 \mu \mathrm{MCQ}$ for $2 \mathrm{~h}$ and then lysted and subjected to SDS-PAGE. (h) CHO cells stably expressing GFP-LC3 were transfected with E46K mutant $\alpha$-synuclein or an empty vector for $48 \mathrm{~h}$ followed by $2 \mathrm{~h}$ of incubation with and without $100 \mu \mathrm{M} \mathrm{CQ}$. GFP fluorescence intensity was assessed using flowcytometry. For all of the graphs, data were shown as means $\pm \mathrm{SD}$ for three independent experiments performed in triplicate. ${ }^{*} P<0.05 ;{ }^{* *} P<0.01 ;{ }^{* * *} P<0.001$ by two-tailed Student's $t$ test 